\title{
NUMERICAL APPROXIMATIONS FOR A NONLOCAL EVOLUTION EQUATION
}

\author{
MAYTE PÉREZ-LLANOS AND JULIO D. ROSSI
}

ABSTRACT. In this paper we study numerical approximations of the nonlocal $p$-Laplacian type diffusion equation,

$$
u_{t}(t, x)=\int_{\Omega} J(x-y)|u(t, y)-u(t, x)|^{p-2}(u(t, y)-u(t, x)) d y
$$

First, we find that a semidiscretization in space of this problem gives rise to an ODE system whose solutions converge uniformly to the continuous one as the mesh size goes to zero. Moreover, the semidiscrete approximation shares some properties of the continuos problem: it preserves the total mass and the solution converges to the mean value of the initial condition as $t$ goes to infinity.

Next, we discretize also the time variable and present a totally discrete method which also enjoys the above mentioned properties.

In addition, we investigate the limit as $p$ goes to infinity in these approximations and obtain a discrete model for the evolution of a sandpile.

Finally, we present some numerical experiments that illustrate our results.

\section{INTRODUCTION}

Our main goal in this paper is to approximate numerically a nonlocal nonlinear diffusion problem, involving the nonlocal p-Laplacian operator (with homogeneous Neumann boundary conditions). More precisely, we deal with the problem

$$
\left\{\begin{array}{l}
u_{t}(t, x)=\int_{\Omega} J(x-y)|u(t, y)-u(t, x)|^{p-2}(u(t, y)-u(t, x)) d y \\
u(x, 0)=u_{0}(x)
\end{array}\right.
$$

being $J: \mathbb{R}^{d} \rightarrow \mathbb{R}$ a nonnegative continuous radial function with compact support verifying $J(0)>0$. We also assume that $\int_{\mathbb{R}^{d}} J(x) d x=1$ to simplify our arguments (although this condition is not necessary to prove our results). We take $1 \leq p<+\infty$ and $\Omega \subset \mathbb{R}^{d}$ a bounded domain. Existence and uniqueness of a strong solution to (1.1) can be found in [3].

Key words and phrases. Numerical approximations, Nonlocal diffusion, $p$-Laplacian, Neumann boundary conditions, Sandpiles.

2000 Mathematics Subject Classification. 65R20, 65N40, 45G10. 
Nonlocal evolution equations of the form

$$
u_{t}(x, t)=J * u-u(x, t)=\int_{\mathbb{R}^{d}} J(x-y)(u(y, t)-u(x, t)) d y,
$$

have many applications in modelizing diffusion processes, see [1], [4], [5], [9], [10], [11], [12], [13], [14], [19], [20], [23], [24], [25], and even in the treatment of images, see [8, 21].

As stated in [19], if $u(x, t)$ represents the density of a single population at the point $x$ at time $t$, and $J(x-y)$ is considered as the probability distribution of jumping from location $y$ to location $x$, then the convolution $(J * u)(x, t)=\int_{\mathbb{R}^{d}} J(y-x) u(y, t) d y$ is the rate at which individuals are arriving to position $x$ from any other place, while $-u(x, t)=$ $-\int_{\mathbb{R}^{d}} J(y-x) u(x, t) d y$ is the rate at which they are leaving location $x$ to travel to any other site. Under these considerations and in the absence of external or internal sources, the density $u$ satisfies equation (1.2). Equation (1.2) is known as a nonlocal diffusion equation since the diffusion of the density $u$ at a point $x$ and time $t$ depends on all the values of $u$ in a neighborhood of $x$, through the convolution term $J * u$.

The nonlocal diffusion equations share many properties with the corresponding local problems. In [3] the authors find that problem (1.1) is the nonlocal analogous problem to the well known local $p$-Laplacian evolution equation $u_{t}=\operatorname{div}\left(|\nabla u|^{p-2} \nabla u\right)$, for $p>1$, (while the extreme case, $p=1$ corresponds to the total variation flow) with homogeneous Neumann boundary conditions. Indeed, if one rescales the kernel $J$ properly, solutions to (1.1) converge to solutions of the local problem.

Note that, since we are integrating in $\Omega$, we are imposing that diffusion takes place only in $\Omega$. There is no flux of individuals across the boundary. Hence, we are dealing here with the nonlocal analogous to Neumann boundary conditions.

Finally, concerning the large time behaviour, solutions to (1.1) converge to the mean value of the initial condition, as it happens for the local problem. Moreover, in [2] the limit as $p \rightarrow \infty$ is considered. It is given by a nonlocal model for the formation and growth of a sandpile, analogous to the local model described in $[16,17,18]$.

However, up to our knowledge, there is none rigorous study of numerical approximations for these kind of problems.

Now, let us describe our results. We look in detail to the one-dimensional case, that is $\Omega=[0, L]$, since the extension to several space dimensions is straightforward. Assume that the support of $J$ is the interval $[-S, S]$. Let us consider a partition (not necessarily uniform) $x_{1}, \cdots, x_{N+1}$ of the interval $[0, L]$ of size $h=\max \left(x_{i}-x_{i-1}\right)$. Then, the numerical approximation to problem (1.1) solves the following system of O.D.Es, for each node $x_{j}$,

$$
\left\{\begin{array}{l}
u_{j}^{\prime}(t)=\sum_{i \in A} J\left(x_{i}-x_{j}\right) h_{i}\left|u_{i}(t)-u_{j}(t)\right|^{p-2}\left(u_{i}(t)-u_{j}(t)\right), \quad t>0, \\
u_{j}(0)=u_{0}\left(x_{j}\right),
\end{array}\right.
$$

for every $j=1, \cdots, N+1$, being $A=\left\{i\right.$ such that $\left.\left|x_{i}-x_{j}\right| \leq S\right\}$. 
Next, we discretize the time variable using the explicit Euler method and we obtain

$$
\left\{\begin{array}{l}
\frac{u_{j}^{k+1}-u_{j}^{k}}{\tau_{k}}=\sum_{i \in A} J\left(x_{i}-x_{j}\right) h_{i}\left|u_{i}^{k}-u_{j}^{k}\right|^{p-2}\left(u_{i}^{k}-u_{j}^{k}\right), \quad k>0, \\
u_{j}^{0}=u_{0}\left(x_{j}\right) .
\end{array}\right.
$$

Note that for these nonlocal models the explicit Euler scheme is well suited, since it does not need a restrictive stability constraint for the time step, as it happens for local problems. This is related to the lack of regularizing effect in nonlocal problems. See Remark 2.9.

Our results concerning the semidiscrete and totally discrete approximations read as follows (see Sections 2.1 and 2.2 for precise statements).

Solutions to the numerical scheme converge uniformly to the continuous solution as the mesh size $h$ (and the time step $\tau$ ) goes to zero. Moreover, the numerical approximations share some properties of the continuous problem: there is a comparison principle, they preserve the symmetry and the total mass of the initial data and they converge to the mean value of the initial condition as $t$ goes to infinity.

We remark that our results also hold when we deal with approximations in a multidimensional domain. The proofs are similar to the one-dimensional case and are left to the reader. See the short paragraph at the end of Section 2.

We also study the limit as $p \rightarrow \infty$ and we prove that solutions to the semidiscrete scheme (1.3) converge to a nonlocal evolution problem, that can be regarded as a semidiscrete approximation of a model for the evolution of a sandpile, see [2]. In the local sandpile models it is assumed that the maximum slope of a sandpile is one (otherwise the sand configuration becomes unstable), see $[16,17,18]$. In the nonlocal sandpile model described in [2] the same restriction on the slope is assumed but with some freedom at short distances (distances shorter than the size of the support of $J$ ), that can measure irregularities of the sand grains.

The rest of the paper is organized as follows: in Section 2 we study the semidiscrete and the totally discrete approximations in one space dimension, in Section 3 we take the limit as $p \rightarrow \infty$ and finally in Section 4 we show some numerical experiments that illustrate our results.

\section{SEmidiscrete AND totally Discrete numerical Methods}

In this section we develop a fully discrete numerical method to approximate problem (1.1), restricting ourselves to one space dimension, that is, $\Omega=[0, L]$, and $\operatorname{supp}(J)=$ $[-S, S]$. We begin by describing our space discretization.

2.1. Semidiscrete scheme. We perform a discretization of the space variable and show the convergence of the semidiscrete scheme and some asymptotic properties of the approximations. 
To this end we introduce some preliminary notation. Let us consider a partition (not necessarily uniform) $x_{1}, \cdots, x_{N+1}$ of the interval $[0, L]$ of size $h\left(h=\max \left(x_{i}-x_{i-1}\right)\right)$ and the corresponding standard piecewise linear finite element space $V_{h}$. Let us denote by $\left\{\phi_{j}\right\}_{1 \leq j \leq N}$ the usual Lagrange basis of $V_{h}$.

We define the semidiscrete approximation,

$$
u_{h}(x, t)=\sum_{j=1}^{N+1} u_{j}(t) \phi_{j}(x)
$$

Where $u_{i}(t)$ is the solution to the following system of O.D.Es

$$
\left\{\begin{array}{l}
u_{j}^{\prime}(t)=\sum_{i \in A} J\left(x_{i}-x_{j}\right) h_{i}\left|u_{i}(t)-u_{j}(t)\right|^{p-2}\left(u_{i}(t)-u_{j}(t)\right), \quad t>0 \\
u_{j}(0)=u_{0}\left(x_{j}\right)
\end{array}\right.
$$

for every $j=1, \cdots, N+1$, being $A=\left\{i\right.$ such that $\left.\left|x_{i}-x_{j}\right| \leq S\right\}$. Note that local existence and uniqueness of solutions to this system is straightforward from the fact that the right hand side of the equation is locally Lipschitz.

We first show that the semidiscrete solutions converge to the continuous solution. To this end we prove that our solutions satisfy a comparison principle.

Definition 2.1. We say that $\bar{U}$ is a supersolution of problem (2.6) if each of its components satisfies

$$
\left\{\begin{array}{l}
\bar{u}_{j}^{\prime}(t) \geq \sum_{i} J\left(x_{i}-x_{j}\right) h_{i}\left|\bar{u}_{i}(t)-\bar{u}_{j}(t)\right|^{p-2}\left(\bar{u}_{i}(t)-\bar{u}_{j}(t)\right), \quad t>0, \\
\bar{u}_{j}^{0} \geq u_{0}\left(x_{j}\right)
\end{array}\right.
$$

for every $x_{i}$ such that $\left|x_{i}-x_{j}\right| \leq S$ and $j=1, \cdots, N+1$. Analogously, $\underline{U}$ is a subsolution of problem (2.6) if it verifies the previous problem with the reverse inequalities.

Lemma 2.2. Let $\bar{U}$ and $\underline{U}$ be a supersolution and a subsolution of problem (2.13), respectively. Then

$$
\bar{U}(t) \geq U(t) \geq \underline{U}(t) .
$$

Proof. By an approximation procedure we restrict ourselves to consider strict inequalities for the supersolution. Indeed, we can take $\bar{u}_{i}(t)+\delta t+\delta(\delta>0)$ for every $i=1, \cdots, N+1$ as a strict supersolution, and take limit as $\delta \rightarrow 0$ at the end. We show that $\bar{U}(t)>U(t)$ by contradiction. Let us assume that there exists a first time $t_{0}$ and a node $j_{0}$ such that $\bar{u}_{j_{0}}\left(t_{0}\right)=u_{j_{0}}\left(t_{0}\right)=a$; then we have

$$
\begin{aligned}
& 0 \geq \bar{u}_{j_{0}}^{\prime}\left(t_{0}\right)-u_{j_{0}}^{\prime}\left(t_{0}\right)> \\
& \sum_{i \in A} h_{i} J\left(x_{i}-x_{j_{0}}\right)\left(\left|\bar{u}_{i}\left(t_{0}\right)-a\right|^{p-2}\left(\bar{u}_{i}\left(t_{0}\right)-a\right)-\left|u_{i}\left(t_{0}\right)-a\right|^{p-2}\left(u_{i}\left(t_{0}\right)-a\right)\right) \geq 0
\end{aligned}
$$

a contradiction. The inequality $U(t) \geq \underline{U}(t)$ can be handled in a similar way. 
Now we are ready to prove the convergence result.

Theorem 2.3. Let $u \in C\left([0, T] ; L^{1}(\Omega)\right) \cap W^{1,1}\left((0, T) ; L^{1}(\Omega)\right)$, be a positive solution to (1.1) and $u_{h}$ the numerical approximation defined by (2.5). Then there exists a constant $K$, such that for every $h$ small enough it holds

$$
\max _{0 \leq t \leq T}\left\{\max _{x \in[0, L]}\left|u(x, t)-u_{h}(x, t)\right|\right\} \leq K h
$$

Proof. Let us denote by $\varepsilon_{j}(t)=u_{j}(t)-v_{j}(t)$ the error vector, where $v_{j}(t)=u\left(x_{j}, t\right)$. It is easy to check that this vector verifies

$$
\begin{aligned}
\varepsilon_{j}^{\prime}= & \sum_{i \in A} J\left(x_{i}-x_{j}\right) h_{i}\left(\left|u_{i}-u_{j}\right|^{p-2}\left(u_{i}-u_{j}\right)-\left|v_{i}-v_{j}\right|^{p-2}\left(v_{i}-v_{j}\right)\right) \\
& +\sum_{i \in A} J\left(x_{i}-x_{j}\right) h_{i}\left|v_{i}-v_{j}\right|^{p-2}\left(v_{i}-v_{j}\right) \\
& \quad-\int_{\Omega} J(y-x)|u(y)-u(x)|^{p-2}(u(y)-u(x)) d y \\
\leq & \sum_{i \in A} J\left(x_{i}-x_{j}\right) h_{i}(p-1)|\eta|^{p-2}\left|\varepsilon_{i}-\varepsilon_{j}\right|+O(h)
\end{aligned}
$$

being $\eta$ an intermediate value between $u_{i}-u_{j}$ and $v_{i}-v_{j}$, resulting from applying the Mean Value Theorem to the first term. The second term is $O(h)$, since each of the approximations to the integrals are $O\left(h^{2}\right)$ and we add them taking into account the nodes laying on the $\operatorname{supp}(J)$.

Therefore, the error vector solves the following problem,

$$
\left\{\begin{array}{l}
\varepsilon_{j}^{\prime}(t)=\sum_{i \in A} J\left(x_{i}-x_{j}\right) h_{i}(p-1)|\eta|^{p-2}\left|\varepsilon_{i}-\varepsilon_{j}\right|+C h, \quad t>0, \\
\varepsilon_{j}(0)=0
\end{array}\right.
$$

Thus, if we consider $\omega_{j}(t)=\omega(t)=C h t$, for every $j=1, \cdots, N+1, \omega$ is a supersolution of problem (2.7). It can be shown in a similar way to Lemma 2.2, that this problem satisfies a comparison principle. Consequently, $\varepsilon_{j}(t) \leq \omega(t) \leq K h$, for every $t \leq T$ and every $j=1, \cdots, N+1$. Here $K=C T$.

Repeating this argument for the vector $-\varepsilon_{j}(t)$ we conclude that

$$
\left|\varepsilon_{j}(t)\right| \leq K h, \quad \text { for every } j=1, \cdots, N+1,
$$

which finishes the proof.

Let us prove now that, as it happens for the continuous problem, if the initial datum is symmetric, the numerical scheme preserves this property.

Lemma 2.4. Let $u_{0}(x)$ be a symmetric initial datum in $[-L, L]$, that is $u_{0}(x)=u_{0}(-x)$, and consider a symmetric partition $x_{i},-N-1 \leq i \leq N+1$, of this interval, i.e. $x_{i}=-x_{-i}$. 
Then, the solution to problem (2.6) is symmetric, that is, it verifies

$$
u_{i}(t)=u_{-i}(t)
$$

Proof. Let us define $\omega_{j}(t)=u_{-j}(t)$. Then, $\omega_{j}$ satisfies

$$
\left\{\begin{array}{l}
\omega_{j}^{\prime}(t)=\sum_{i \in \bar{A}} J\left(x_{i}-x_{-j}\right) h_{i}\left|u_{i}(t)-\omega_{j}(t)\right|^{p-2}\left(u_{i}(t)-\omega_{j}(t)\right), \quad t>0 \\
\omega_{j}(0)=u_{0}\left(x_{-j}\right)=u_{0}\left(x_{j}\right)
\end{array}\right.
$$

for every $j=-1-N, \cdots, N+1$, being $\bar{A}=\left\{i\right.$ such that $\left.\left|x_{i}-x_{-j}\right| \leq S\right\}$. But note that $\bar{A}=-A$ where $A=\left\{i\right.$ such that $\left.\left|x_{i}-x_{j}\right| \leq S\right\}$ since the partition is symmetric. Taking into account that $J$ is symmetric, we have that the previous equation can be written as follows

$$
\begin{aligned}
\omega_{j}^{\prime}(t) & =\sum_{i \in A} J\left(x_{-i}-x_{-j}\right) h_{i}\left|\omega_{i}(t)-\omega_{j}(t)\right|^{p-2}\left(\omega_{i}(t)-\omega_{j}(t)\right) \\
& =\sum_{i \in A} J\left(x_{i}-x_{j}\right) h_{i}\left|\omega_{i}(t)-\omega_{j}(t)\right|^{p-2}\left(\omega_{i}(t)-\omega_{j}(t)\right) .
\end{aligned}
$$

We conclude the proof by uniqueness of the solutions to the ODE system.

We show that the solutions of the discrete nonlocal problem converge to the mean value of the initial condition. To carry out this task, we prove the following Poincaré's type inequality.

Lemma 2.5. Let $p \geq 1$. Then,

$$
I_{p}=I(J, d, p)=\inf _{\left\{v \in \mathbb{R}^{N+1}: \sum_{i=1}^{N+1} h_{i} v_{i}(t)=0\right\}} \frac{\frac{1}{2} \sum_{i, j=1}^{N} h_{i} h_{j} J\left(x_{i}-x_{j}\right)\left|v_{i}-v_{j}\right|^{p}}{\sum_{j=1}^{N+1} h_{j}\left|v_{j}\right|^{p}}
$$

is strictly positive. In consequence,

$$
I_{p} \sum_{j=1}^{N+1} h_{j}\left|v_{j}-\frac{1}{L} \sum_{i=1}^{N+1} h_{i} v_{i}\right|^{p} \leq \frac{1}{2} \sum_{i, j=1}^{N+1} h_{i} h_{j} J\left(x_{i}-x_{j}\right)\left|v_{i}-v_{j}\right|^{p}
$$

for every $v \in \mathbb{R}^{N+1}$.

Proof. To show (2.8) it suffices to see that for each $v \in \mathbb{R}^{N+1}$ there exists a constant $c>0$ such that

$$
\sum_{j=1}^{N+1} h_{j}\left|v_{j}\right|^{p} \leq c\left(\sum_{i, j=1}^{N+1} h_{i} h_{j} J\left(x_{i}-x_{j}\right)\left|v_{i}-v_{j}\right|^{p}+\left|\sum_{i=1}^{N+1} h_{i} v_{i}\right|\right) .
$$


Arguing by contradiction, if (2.10) does not hold, then for every $n \in \mathbb{N}$ there exists $v_{n} \in$ $\mathbb{R}^{N+1}$ with $\sum_{i=1}^{N+1} h_{i}\left|\left(v_{n}\right)_{i}\right|^{p}=1$, satisfying

$$
1>n\left(\sum_{i, j=1}^{N+1} h_{i} h_{j} J\left(x_{i}-x_{j}\right)\left|\left(v_{n}\right)_{i}-\left(v_{n}\right)_{j}\right|^{p}+\left|\sum_{i=1}^{N+1} h_{i}\left(v_{n}\right)_{i}\right|\right) .
$$

In particular, this implies that $\lim _{n \rightarrow \infty} \sum_{i=1}^{N+1} h_{i}\left(v_{n}\right)_{i}=0$, which contradicts the fact that $\sum_{i} h_{i}\left|\left(v_{n}\right)_{i}\right|^{p}=1$. Note that (2.9) follows immediately from (2.8), for $v_{j}=\omega_{j}-$ $\frac{1}{L} \sum_{i=1}^{N+1} h_{i} \omega_{i}$, for any $\omega \in \mathbb{R}^{N+1}$.

We are now ready to study the asymptotic behaviour of the discrete solutions.

Theorem 2.6. Let $p \geq 1$ and $u_{h} \in V_{h}$ the solution to problem (2.6) corresponding to an initial datum, $u_{0}$. Then, there exists a positive constant $C$, independent of $t$, such that

$$
\sum_{j=1}^{N+1} h_{j}\left|u_{j}(t)-\bar{u}_{0}\right|^{p} \leq C \frac{\sum_{j=1}^{N+1} h_{j}\left|u_{j}(0)\right|^{2}}{t} \rightarrow 0, \quad \text { as } t \rightarrow \infty,
$$

where $\bar{u}_{0}=\frac{1}{L} \sum_{i} h_{i} u_{i}(0)<\infty$.

Proof. We define $\omega_{j}(t)=u_{j}(t)-\bar{u}_{0}$. From (2.6) it is easy to see that $\omega$ verifies

$$
\begin{aligned}
\frac{d}{d t} \sum_{j=1}^{N} h_{j}\left|\omega_{j}\right|^{p}= & p \sum_{j=1}^{N+1} h_{j}\left|\omega_{j}\right|^{p-2} \omega_{j} \sum_{i \in A} h_{i} J\left(x_{i}-x_{j}\right)\left|\omega_{i}-\omega_{j}\right|^{p-2}\left(\omega_{i}-\omega_{j}\right) \\
= & -p \sum_{j=1}^{N+1} \sum_{i \in A} h_{j} h_{i} J\left(x_{i}-x_{j}\right)\left(\left|\omega_{i}\right|^{p-2} \omega_{i}-\left|\omega_{j}\right|^{p-2} \omega_{j}\right)\left|\omega_{i}-\omega_{j}\right|^{p-2}\left(\omega_{i}-\omega_{j}\right) \\
& +p \sum_{j=1}^{N+1} \sum_{i \in A} h_{j} h_{i} J\left(x_{i}-x_{j}\right)\left|\omega_{i}\right|^{p-2} \omega_{i}\left|\omega_{i}-\omega_{j}\right|^{p-2}\left(\omega_{i}-\omega_{j}\right) \\
= & -\frac{p}{2} \sum_{j=1}^{N+1} \sum_{i \in A} h_{j} h_{i} J\left(x_{i}-x_{j}\right)\left(\left|\omega_{i}\right|^{p-2} \omega_{i}-\left|\omega_{j}\right|^{p-2} \omega_{j}\right)\left|\omega_{i}-\omega_{j}\right|^{p-2}\left(\omega_{i}-\omega_{j}\right) .
\end{aligned}
$$

Therefore, $\sum_{j=1}^{N+1} h_{j}\left|\omega_{j}\right|^{p}$ is non increasing.

On the other hand, note that adding the equation in $(2.6)_{1}$ in all of the nodes, we obtain

$$
\begin{aligned}
\sum_{j=1}^{N+1} h_{j} u_{j}^{\prime}(t) & =\sum_{j=1}^{N+1} \sum_{i \in A} h_{j} J\left(x_{i}-x_{j}\right) h_{i}\left|u_{i}(t)-u_{j}(t)\right|^{p-2}\left(u_{i}(t)-u_{j}(t)\right) \\
& =\frac{1}{2} \sum_{j=1}^{N+1} \sum_{i \in A} h_{j} J\left(x_{i}-x_{j}\right) h_{i}\left|u_{i}(t)-u_{j}(t)\right|^{p-2}\left(u_{i}(t)-u_{j}(t)\right) \\
& +\frac{1}{2} \sum_{i=1}^{N+1} \sum_{j \in A} h_{j} J\left(x_{i}-x_{j}\right) h_{i}\left|u_{i}(t)-u_{j}(t)\right|^{p-2}\left(u_{i}(t)-u_{j}(t)\right)=0 .
\end{aligned}
$$


That is, the mass is preserved, $\frac{1}{L} \sum_{j=1}^{N+1} h_{j} u_{j}(t)=\bar{u}_{0}$, for every $t \geq 0$ and, consequently, $\frac{1}{L} \sum_{j=1}^{N+1} h_{j} \omega_{j}(t)=0$, for every $t \geq 0$. Thus we can apply to this function the Poincare's inequality (2.9) and we obtain

$$
I_{p} \sum_{j=1}^{N+1} h_{j}\left|\omega_{j}\right|^{p} \leq \frac{1}{2} \sum_{i, j=1}^{N+1} h_{i} h_{j} J\left(x_{i}-x_{j}\right)\left|\omega_{i}-\omega_{j}\right|^{p},
$$

from which it easily follows,

$$
\begin{aligned}
t \sum_{j=1}^{N+1} h_{j}\left|\omega_{j}(t)\right|^{p} & \leq \int_{0}^{t} \sum_{j=1}^{N+1} h_{j}\left|\omega_{j}(s)\right|^{p} d s \\
& \leq C \int_{0}^{t} \sum_{i, j=1}^{N+1} h_{i} h_{j} J\left(x_{i}-x_{j}\right)\left|\omega_{i}(s)-\omega_{j}(s)\right|^{p} d s \\
& =C \int_{0}^{t} \sum_{i, j=1}^{N+1} h_{i} h_{j} J\left(x_{i}-x_{j}\right)\left|u_{i}(s)-u_{j}(s)\right|^{p} d s .
\end{aligned}
$$

Now, we multiply the equation $(2.6)_{1}$ by $u_{j}$ and we add in all the nodes, it is easy to check that

$$
\begin{aligned}
\frac{1}{2} \frac{d}{d t}\left(\sum_{j=1}^{N+1} h_{j}\left|u_{j}(t)\right|^{2}\right)= & \sum_{i, j=1}^{N+1} J\left(x_{i}-x_{j}\right) h_{i} h_{j}\left|u_{i}(t)-u_{j}(t)\right|^{p-2}\left(u_{i}(t)-u_{j}(t)\right) u_{j}(t) \\
= & -\sum_{i, j=1}^{N+1} J\left(x_{i}-x_{j}\right) h_{i} h_{j}\left|u_{i}(t)-u_{j}(t)\right|^{p} \\
& +\sum_{i, j=1}^{N+1} J\left(x_{i}-x_{j}\right) h_{i} h_{j}\left|u_{i}(t)-u_{j}(t)\right|^{p-2}\left(u_{i}(t)-u_{j}(t)\right) u_{i}(t) \\
= & -\frac{1}{2} \sum_{i, j=1}^{N+1} J\left(x_{i}-x_{j}\right) h_{i} h_{j}\left|u_{i}(t)-u_{j}(t)\right|^{p}
\end{aligned}
$$

Integrating in time, we deduce that

$$
\sum_{j=1}^{N+1} h_{j}\left|u_{j}(t)\right|^{2}-\sum_{j=1}^{N+1} h_{j}\left|u_{j}(0)\right|^{2}=-\int_{0}^{t} \sum_{i, j=1}^{N+1} h_{i} h_{j} J\left(x_{i}-x_{j}\right)\left|u_{i}(s)-u_{j}(s)\right|^{p} d s .
$$

Thus,

$$
\int_{0}^{t} \sum_{i, j=1}^{N+1} h_{i} h_{j} J\left(x_{i}-x_{j}\right)\left|u_{i}(s)-u_{j}(s)\right|^{p} d s \leq \sum_{j=1}^{N+1} h_{j}\left|u_{j}(0)\right|^{2},
$$

which plugged into (2.12) gives the desired conclusion, (2.11). 
2.2. A fully discrete scheme. Now we perform the discretization in time, using the explicit Euler method, that is

$$
\left\{\begin{array}{l}
\frac{u_{j}^{k+1}-u_{j}^{k}}{\tau_{k}}=\sum_{i \in A} J\left(x_{i}-x_{j}\right) h_{i}\left|u_{i}^{k}-u_{j}^{k}\right|^{p-2}\left(u_{i}^{k}-u_{j}^{k}\right), \quad k>0, \\
u_{j}^{0}=u_{0}\left(x_{j}\right)
\end{array}\right.
$$

being $A=\left\{i:\left|x_{i}-x_{j}\right| \leq S\right\}$, for every $j=1, \cdots, N+1$. We denote by $U^{k}=$ $\left(u_{1}^{k}, \cdots, u_{N+1}^{k}\right)$ the vector whose components solve the previous system (2.13).

The symmetry property given in Lemma 2.4 follows similarly from reflection and uniqueness for the totally discrete method. However, the comparison principle for problem (2.13) requires a condition on the time step $\tau_{k}$, which is not restrictive. In order to show this comparison principle we give the following definitions.

Definition 2.7. We say that $\bar{U}^{k}$ is a supersolution of problem (2.13) if each of its components satisfies

$$
\left\{\begin{array}{l}
\frac{\bar{u}_{j}^{k+1}-\bar{u}_{j}^{k}}{\tau_{k}} \geq \sum_{i \in A} J\left(x_{i}-x_{j}\right) h_{i}\left|z_{i}^{k}-z_{j}^{k}\right|^{p-2}\left(\bar{u}_{i}^{k}-\bar{u}_{j}^{k}\right), \quad k>0, \\
\bar{u}_{j}^{0} \geq u_{0}\left(x_{j}\right)
\end{array}\right.
$$

being $A=\left\{i:\left|x_{i}-x_{j}\right| \leq S\right\}$, for every $j=1, \cdots, N+1$. Analogously, $\underline{U}^{k}$ is a subsolution of problem (2.13) if it verifies (2.14) with the reverse inequalities.

Proposition 2.8. Let $\bar{U}^{k}$ and $\underline{U}^{k}$ a super and a subsolution of problem (2.13), respectively, such that $\bar{U}^{0} \leq \underline{U}^{0}$. If the time step verifies

$$
\tau_{k}<\frac{1}{2(p-1) \max _{j}\left|\bar{u}_{j}^{k}\right|^{p-2}},
$$

then $\bar{U}^{k} \leq \underline{U}^{k}$, for every $k>0$.

Proof. We define $Z^{k}=\bar{U}^{k}-\underline{U}^{k}$. Note that, as before, by an approximation argument, we can assume in (2.14) strict inequalities. Therefore, $Z^{k}$ verifies for each $j=1, \cdots, N+1$ and every $k>0$ the following system

$$
\left\{\begin{array}{l}
\frac{z_{j}^{k+1}-z_{j}^{k}}{\tau_{k}}>\sum_{i \in A} J\left(x_{i}-x_{j}\right) h_{i}\left(\left|\bar{u}_{i}^{k}-\bar{u}_{j}^{k}\right|^{p-2}\left(\bar{u}_{i}^{k}-\bar{u}_{j}^{k}\right)-\left|\underline{u}_{i}^{k}-\underline{u}_{j}^{k}\right|^{p-2}\left(\underline{u}_{i}^{k}-\underline{u}_{j}^{k}\right)\right), \\
z_{j}^{0}>0 .
\end{array}\right.
$$

Let us argue by contradiction and suppose that there exists a first time $t^{k+1}$ and a node $x_{j}$ such that $z_{j}^{k+1} \leq 0$ while $z_{j}^{k}>0$. From the corresponding equation for that node we obtain 


$$
\begin{aligned}
z_{j}^{k+1} & >z_{j}^{k}+\tau_{j} \sum_{i} J\left(x_{i}-x_{j}\right) h_{i}\left(\left|\bar{u}_{i}^{k}-\bar{u}_{j}^{k}\right|^{p-2}\left(\bar{u}_{i}^{k}-\bar{u}_{j}^{k}\right)-\left|\underline{u}_{i}^{k}-\underline{u}_{j}^{k}\right|^{p-2}\left(\underline{u}_{i}^{k}-\underline{u}_{j}^{k}\right)\right) \\
& =z_{j}^{k}+(p-1) \tau_{j} \sum_{i} J\left(x_{i}-x_{j}\right) h_{i}\left|\theta_{i, j}^{k}\right|^{p-2}\left(z_{i}^{k}-z_{j}^{k}\right),
\end{aligned}
$$

being $\theta_{i, j}^{k}$ a value between $\bar{u}_{i}^{k}-\bar{u}_{j}^{k}$ and $\underline{u}_{i}^{k}-\underline{u}_{j}^{k}$. Let $\eta_{j}^{k}=\max _{i}\left\{\theta_{i, j}^{k}\right\}$. Neglecting the positive terms in the previous inequality and taking (2.15) into account we get

$$
\begin{aligned}
z_{j}^{k+1} & >z_{j}^{k}\left(1-(p-1) \tau_{j}\left|\eta_{j}^{k}\right|^{p-2} \sum_{i} J\left(x_{i}-x_{j}\right) h_{i}\right) \\
& =z_{j}^{k}\left(1-(p-1) \tau_{j}\left|\eta_{j}^{k}\right|^{p-2}(1+O(h))\right) \geq 0
\end{aligned}
$$

which is a contradiction.

Remark 2.9. Note that the condition (2.15) does not depend on $h$. This has to be contrasted with the analogous condition for the local problem. Indeed, the condition ensuring the comparison principle for an analogous numerical scheme (based on a discretization in space using piecewise finite element with mass lumping and the Euler explicit method in the time variable) for the corresponding local problem is

$$
\tau_{k} \leq h^{p} /\left(2(p-1) \max _{j}\left|u_{j}^{k}\right|^{p-2}\right)
$$

for a uniform mesh of size $h$.

We prove now the convergence result for this numerical scheme.

Theorem 2.10. Let $u \in C\left([0, T] ; L^{1}(\Omega)\right) \cap W^{1,1}\left((0, T) ; L^{1}(\Omega)\right)$, be a positive solution to (1.1) and $u_{h}^{k}$ the numerical approximation solving problem (2.13). Then there exists a constant $K$, such that for every $h$ small enough it holds

$$
\max _{0 \leq t_{k} \leq T}\left\{\max _{x \in[0, L]}\left|u\left(x, t_{k}\right)-u_{h}^{k}\right|\right\} \leq K(h+\tau)
$$

being $h=\max _{i} h_{i}$ and $\tau=\max _{k} \tau_{k}$. 
Proof. We define the error vector at time $t^{k+1}$ as $\varepsilon_{j}^{k+1}=u_{j}^{k+1}-v_{j}^{k+1}$, being $v_{j}^{k+1}=$ $u\left(x_{j}, t_{k+1}\right)$. It verifies the following problem

$$
\begin{aligned}
\left|\frac{\varepsilon_{j}^{k+1}-\varepsilon_{j}^{k}}{\tau_{k}}\right|= & \mid \sum_{i \in A} J\left(x_{i}-x_{j}\right) h_{i}\left(\left|u_{i}^{k}-u_{j}^{k}\right|^{p-2}\left(u_{i}^{k}-u_{j}^{k}\right)-\left|v_{i}^{k}-v_{j}^{k}\right|^{p-2}\left(v_{i}^{k}-v_{j}^{k}\right)\right) \\
& +\sum_{i \in A} J\left(x_{i}-x_{j}\right) h_{i}\left|v_{i}^{k}-v_{j}^{k}\right|^{p-2}\left(v_{i}^{k}-v_{j}^{k}\right) \\
& -\frac{1}{\tau_{k}} \int_{t_{k}}^{t_{k+1}} \int_{\Omega} J(x-y)|u(y, s)-u(x, s)|^{p-2}(u(y, s)-u(x, s)) d y d s \mid \\
\leq & \sum_{i \in A} J\left(x_{i}-x_{j}\right) h_{i}(p-1)|\eta|^{p-2}\left|\varepsilon_{i}^{k}-\varepsilon_{j}^{k}\right|+O(h+\tau),
\end{aligned}
$$

where, as before, $\eta$ comes from the Mean Value Theorem. Therefore,

$$
\left|\frac{\varepsilon_{j}^{k+1}-\varepsilon_{j}^{k}}{\tau_{k}}\right| \leq \sum_{i \in A} J\left(x_{i}-x_{j}\right) h_{i}(p-1)|\eta|^{p-2}\left|\varepsilon_{i}^{k}-\varepsilon_{j}^{k}\right|+C(h+\tau) .
$$

Analogously as before, if we consider $\omega_{j}^{k}=\omega^{k}=C k \tau_{k}(h+\tau)$, for every $j, k$, then $\omega_{j}^{k}$ is a supersolution of the previous problem. Notice that there exists $k_{0}$ such that $t_{k_{0}} \geq T$, thus take $K=C t_{k_{0}}$. The end of the proof follows again by comparison and applying the same reasoning to $-\varepsilon_{j}^{k}$.

We conclude the study of the totally discrete method by showing that these solutions also preserve the total mass and tend to the mean value of the initial datum, as it happened for the semidiscrete case. Nevertheless, we cannot prove now the rate of such convergence in terms of $t$.

Theorem 2.11. Let $u_{j}^{k}$ be the solution to problem (2.13) and $\bar{u}_{0}=\frac{1}{L} \sum_{i} h_{i} u_{i}(0)$, the mean value of the initial condition. Then, the total mass is preserved,

$$
\frac{1}{L} \sum_{j} h_{j} u_{j}^{k}=\bar{u}_{0} \quad \forall k .
$$

Moreover,

$$
\max _{j} u_{j}^{k}-\bar{u}_{0} \longrightarrow 0, \text { as } k \text { increases. }
$$

Proof. To show the conservation of the mass we sum the equation $(2.13)_{1}$ in all of the nodes,

$$
\sum_{j=1}^{N+1} \frac{u_{j}^{k+1}-u_{j}^{k}}{\tau_{k}}=\sum_{j=1}^{N+1} \sum_{i \in A} J\left(x_{i}-x_{j}\right) h_{i}\left|u_{i}^{k}-u_{j}^{k}\right|^{p-2}\left(u_{i}^{k}-u_{j}^{k}\right)=0,
$$

which vanishes by the same reasoning as in the semidiscrete case. Therefore,

$$
\sum_{j=1}^{N+1} u_{j}^{k+1}=\sum_{j=1}^{N+1} u_{j}^{k}, \quad \text { for all } k \geq 0 .
$$


To prove the second statement we begin with the study of the symmetric case. Let us consider a symmetric initial condition with a unique maximum at the central node. Since the scheme preserves this property, the discrete solution will attain this maximum at the central node for every $k$. Denote by $x_{j_{0}}$ such a node and define $\omega_{j}^{k}=u_{j}^{k}-\bar{u}_{0}$. Then, $\omega_{j 0}^{k}=\max _{j} \omega_{j}^{k}$ and it verifies the same equations than $u_{j}^{k}$. Recalling the equation for the node $x_{j_{0}}$,

$$
\omega_{j_{0}}^{k+1}=\omega_{j_{0}}^{k}+\tau_{k}\left(\sum_{i} J\left(x_{i}-x_{j_{0}}\right) h_{i}\left|\omega_{i}^{k}-\omega_{j_{0}}^{k}\right|^{p-2}\left(\omega_{i}^{k}-\omega_{j_{0}}^{k}\right)\right),
$$

We show that $\omega_{j_{0}}^{k}$ has to decrease strictly as $k$ increases. Arguing by contradiction, let us suppose that the value for this node remains the same from some time $t^{k}$. Consider the step $k+1$. Note that, since $\omega_{j_{0}}^{k}$ is the node at which $\omega_{j}^{k}$ attains its maximum at time $t^{k}$, the term of the sum is non positive. Thus, if $\omega_{j_{0}}^{k+1}=\omega_{j_{0}}^{k}$ this term is zero. Consequently, $\omega_{l}^{k}=\omega_{j_{0}}^{k}$ for every $x_{l} \in\left[x_{j_{0}}-S, x_{j_{0}}+S\right]$.

If $\omega_{j_{0}}^{k+2}=\omega_{j_{0}}^{k+1}$ we deduce as before that $\omega_{l}^{k+1}=\omega_{j_{0}}^{k+1}$ for every $x_{l} \in\left[x_{j_{0}}-S, x_{j_{0}}+S\right]$. But those values where maxima at step $k$, that remain being the same in the following step. Therefore, applying the same reasoning in these nodes, we get that $\omega_{l}^{k+1}=\omega_{j_{0}}^{k+1}$ for every $x_{l} \in\left[x_{j_{0}}-2 S, x_{j_{0}}+2 S\right]$. In a number of steps $[L / S]+1$ we get a contradiction.

For the general case, the node at which the discrete solution attains its maximum could vary with $k$. Denote by $x_{j_{1}}^{k+1}$ the node at which $\omega_{j_{1}}^{k+1}=\max _{j} \omega_{j}^{k+1}$. But we can take the time steps $\tau_{k}$ small enough, so as to assure that the distance between these two nodes, $\left|x_{j_{0}}-x_{j_{1}}\right| \leq S$, concluding the proof similarly to the symmetric case.

To deal with the same problem in several space dimensions, let $\Omega$ be a bounded domain in $\mathbb{R}^{d}$ and let $x_{1}, \ldots, x_{N+1}$ be a set of points in $\Omega$ that are uniformly distributed (by this we mean that in every ball of radius $S$ in $\Omega$ there are at least $r \sim|\Omega| / S^{N}$ points). Then we can discretize as before imposing that the values of $U$ at the nodes $x_{i}$ verify the ODE system (2.5) (or the totally discrete system (2.13)). The extension of our results to this setting is easy. Indeed, comparison arguments are shown in the same way as for the 1-d case and the convergence result follows by the same arguments as before, once one notices that it holds that

$$
\int_{\Omega} J\left(x_{j}-y\right) u(y) d y \sim|B(0, S)| \frac{1}{r} \sum_{i \in A} J\left(x_{i}-x_{j}\right) u\left(x_{j}\right),
$$

as the number of points goes to infinity $(N \rightarrow \infty)$.

\section{The LiMit AS $p \rightarrow \infty$}

To identify the limit as $p \rightarrow \infty$ of the solutions $u_{p, h}$ of the semidiscrete problem (1.3) we will use the methods of convex analysis, and so we first recall some terminology (see [15] 
and [6]). If $H$ is a real Hilbert space with inner product $($,$) and \Psi: H \rightarrow(-\infty,+\infty]$ is convex, then the subdifferential of $\Psi$ is defined as the multivalued operator $\partial \Psi$ given by

$$
v \in \partial \Psi(u) \Longleftrightarrow \Psi(w)-\Psi(u) \geq(v, w-u) \quad \forall w \in H .
$$

The epigraph of $\Psi$ is defined by $\operatorname{Epi}(\Psi)=\{(u, \lambda) \in H \times \mathbb{R}: \lambda \geq \Psi(u)\}$.

Given $K$ a closed convex subset of $H$, the indicator function of $K$ is defined by

$$
I_{K}(u)= \begin{cases}0 & \text { if } u \in K, \\ +\infty & \text { if } u \notin K .\end{cases}
$$

Then it is easy to see that the subdifferential is characterized as follows,

$$
v \in \partial I_{K}(u) \Longleftrightarrow u \in K \text { and }(v, w-u) \leq 0 \quad \forall w \in K
$$

In case the convex functional $\Psi: H \rightarrow(-\infty,+\infty]$ is proper, lower-semicontinuous and $\min \Psi=0$, it is well known (see [6]) that the abstract Cauchy problem

$$
\left\{\begin{array}{l}
u^{\prime}(t)+\partial \Psi(u(t)) \ni f(t), \quad \text { a.e } t \in(0, T), \\
u(0)=u_{0}
\end{array}\right.
$$

has a unique strong solution for any $f \in L^{2}(0, T ; H)$ and $u_{0} \in \overline{D(\partial \Psi)}$.

The following convergence was studied by Mosco in [22]. Suppose $X$ is a metric space and $A_{n} \subset X$. We define

$$
\liminf _{n \rightarrow \infty} A_{n}=\left\{x \in X: \exists x_{n} \in A_{n}, x_{n} \rightarrow x\right\}
$$

and

$$
\limsup _{n \rightarrow \infty} A_{n}=\left\{x \in X: \exists x_{n_{k}} \in A_{n_{k}}, x_{n_{k}} \rightarrow x\right\} .
$$

In the case $X$ is a normed space, we note by $s-\lim$ and $w$ - lim the above limits associated respectively to the strong and to the weak topology of $X$.

Given a sequence $\Psi_{n}, \Psi: H \rightarrow(-\infty,+\infty]$ of convex lower-semicontinuous functionals, we say that $\Psi_{n}$ converges to $\Psi$ in the sense of Mosco if

$$
w-\limsup _{n \rightarrow \infty} \operatorname{Epi}\left(\Psi_{n}\right) \subset \operatorname{Epi}(\Psi) \subset s-\liminf _{n \rightarrow \infty} \operatorname{Epi}\left(\Psi_{n}\right) .
$$

As consequence of the results in [7] we can write the following result.

Theorem 3.1. Let $\Psi_{n}, \Psi: H \rightarrow(-\infty,+\infty]$ convex lower-semicontinuous functionals. Then the following statements are equivalent:

(i) $\Psi_{n}$ converges to $\Psi$ in the sense of Mosco.

(ii) $\left(I+\lambda \partial \Psi_{n}\right)^{-1} u \rightarrow(I+\lambda \partial \Psi)^{-1} u, \quad \forall \lambda>0, u \in H$.

Moreover, any of these two conditions $(i)$ or (ii) imply that 
(iii) for every $u_{0} \in \overline{D(\partial \Psi)}$ and $u_{0, n} \in \overline{D\left(\partial \Psi_{n}\right)}$ such that $u_{0, n} \rightarrow u_{0}$, and every $f_{n}, f \in$ $L^{2}(0, T ; H)$ with $f_{n} \rightarrow f$, if $u_{n}(t), u(t)$ are the strong solutions of the abstract Cauchy problems

$$
\left\{\begin{array}{l}
u_{n}^{\prime}(t)+\partial \Psi_{n}\left(u_{n}(t)\right) \ni f_{n}, \quad \text { a.e. } t \in(0, T), \\
u_{n}(0)=u_{0, n}
\end{array}\right.
$$

and

$$
\left\{\begin{array}{l}
u^{\prime}(t)+\partial \Psi(u(t)) \ni f, \quad \text { a.e. } t \in(0, T) \\
u(0)=u_{0}
\end{array}\right.
$$

respectively, then

$$
u_{n} \rightarrow u \quad \text { in } C([0, T]: H)
$$

3.1. Limit as $p \rightarrow \infty$. Let us consider the numerical semidiscrete approximations of the nonlocal $p$-Laplacian evolution problem with a source given by $f=\left(f_{1}, \ldots, f_{N+1}\right)$, $f_{i}=f\left(x_{i}\right)$,

$$
\left\{\begin{array}{l}
u_{j}^{\prime}(t)=\sum_{i \in A} J\left(x_{i}-x_{j}\right) h_{i}\left|u_{i}(t)-u_{j}(t)\right|^{p-2}\left(u_{i}(t)-u_{j}(t)\right)+f_{j}(t), \quad t>0, \\
u_{j}(0)=u_{0}\left(x_{j}\right)
\end{array}\right.
$$

for every $j=1, \cdots, N+1$, being $A=\left\{i\right.$ such that $\left.\left|x_{i}-x_{j}\right| \leq S\right\}$.

This problem is associated to the energy functional

$$
G_{p}(v)=\frac{1}{2 p} \sum_{i} \sum_{j} J\left(x_{i}-x_{j}\right) h_{i} h_{j}\left|v_{i}-v_{j}\right|^{p}
$$

in the Hilbert space $H=\mathbb{R}^{N+1}$. Note that $G_{p}$ is differentiable, hence the subdifferential is the usual derivative.

With a formal calculation, taking limit as $p \rightarrow \infty$, we arrive to the functional (recall that $J$ is supported in $B(0, S)$ )

$$
G_{\infty}(v)= \begin{cases}0 & \text { if }\left|v_{i}-v_{j}\right| \leq 1, \text { for }\left|x_{i}-x_{j}\right|<S \\ +\infty & \text { in other case }\end{cases}
$$

Hence, if we define

$$
K_{\infty}:=\left\{v \in \mathbb{R}^{N+1}:\left|v_{i}-v_{j}\right| \leq 1, \text { for }\left|x_{i}-x_{j}\right|<S\right\},
$$

we have that the functional $G_{\infty}$ is determined by the indicator function of the set $K_{\infty}$. Then, the nonlocal semidiscrete limit problem can be written as

$$
\left\{\begin{array}{l}
f(t)-U^{\prime}(t) \in \partial I_{K_{\infty}}(U(t)), \quad \text { a.e. } t \in(0, T), \\
u_{j}(0)=u_{0}\left(x_{j}\right)
\end{array}\right.
$$


Theorem 3.2. Let $T>0$, and an initial condition $u_{0}$ such that $\left|u_{0}(x)-u_{0}(y)\right| \leq 1$, for $|x-y|<S$, and $u_{p, h}$ the unique solution to (3.18). Then, if $U$ is the unique solution to (3.19), we have

$$
\lim _{p \rightarrow \infty} \sup _{t \in[0, T]}\left|u_{p, h}(t)-U(t)\right|=0 .
$$

Proof of Theorem 3.2. Let $T>0$. By Theorem 3.1, to prove the result it is enough to show that the functionals

$$
G_{p}(v)=\frac{1}{2 p} \sum_{i} \sum_{j} J\left(x_{i}-x_{j}\right) h_{i} h_{j}\left|v_{i}-v_{j}\right|^{p}
$$

converge to

$$
G_{\infty}(v)= \begin{cases}0 & \text { if }\left|v_{i}-v_{j}\right| \leq 1, \text { for }\left|x_{i}-x_{j}\right|<S \\ +\infty & \text { in other case, }\end{cases}
$$

as $p \rightarrow \infty$, in the sense of Mosco. Note that in (3.17) weak and strong convergences are the same since we have $H=\mathbb{R}^{N+1}$.

First, let us check that

$$
\operatorname{Epi}\left(G_{\infty}\right) \subset \liminf _{p \rightarrow \infty} \operatorname{Epi}\left(G_{p}\right) .
$$

To this end let us consider $(U, \lambda) \in \operatorname{Epi}\left(G_{\infty}\right)$. We can assume that $U \in K_{\infty}$ and $\lambda \geq 0$ (since $G_{\infty}(U)=0$ ). Now take for each $p$

$$
V_{p}=U \quad \text { and } \quad \lambda_{p}=G_{p}(U)+\lambda .
$$

Then, since $\lambda \geq 0$ we have $\left(V_{p}, \lambda_{p}\right) \in \operatorname{Epi}\left(G_{p}\right)$. It is obvious that $V_{p}=U \rightarrow U$ in $\mathbb{R}^{N+1}$, and moreover, since $U \in K_{\infty}$ then $\left|u_{i}-u_{j}\right| \leq 1$, for $\left|x_{i}-x_{j}\right|<S$ and we get

$$
G_{p}(U)=\frac{1}{2 p} \sum_{i} \sum_{j} J\left(x_{i}-x_{j}\right) h_{i} h_{j}\left|u_{i}-u_{j}\right|^{p} \leq \frac{1}{2 p} \sum_{i} \sum_{j} J\left(x_{i}-x_{j}\right) h_{i} h_{j} \rightarrow 0
$$

as $p \rightarrow \infty$. Therefore, $\lambda_{p} \rightarrow \lambda$ as $p \rightarrow \infty$ and we get (3.20).

Finally, let us prove that

$$
\limsup _{p \rightarrow \infty} \operatorname{Epi}\left(G_{p}\right) \subset \operatorname{Epi}\left(G_{\infty}\right) .
$$

To this end, let us consider a sequence $\left(U_{p_{j}}, \lambda_{p_{j}}\right) \in \operatorname{Epi}\left(G_{p_{j}}\right)$, that is, $G_{p_{j}}\left(U_{p_{j}}\right) \leq \lambda_{p_{j}}$, with $U_{p_{j}} \rightarrow U$, and $\lambda_{p_{j}} \rightarrow \lambda$. Therefore, we have that $\lambda \geq 0$, since $0 \leq G_{p_{j}}\left(U_{p_{j}}\right) \leq \lambda_{p_{j}} \rightarrow \lambda$. On the other hand,

$$
\lambda+1 \geq G_{p_{k}}\left(U_{p_{k}}\right)=\frac{1}{2 p_{k}} \sum_{i} \sum_{j} J\left(x_{i}-x_{j}\right) h_{i} h_{j}\left|\left(u_{p_{k}}\right)_{i}-\left(u_{p_{k}}\right)_{j}\right|^{p_{k}} .
$$

Since any term in the above sum is nonnegative, we conclude that

$$
\lambda+1 \geq \frac{1}{2 p_{k}} h_{i} h_{j} J\left(x_{i}-x_{j}\right)\left|\left(u_{p_{k}}\right)_{i}-\left(u_{p_{k}}\right)_{j}\right|^{p_{k}}
$$


for some $i, j$ such that $\left|x_{i}-x_{j}\right| \leq S$. Hence, since $\left(u_{p_{k}}\right)_{i} \rightarrow u_{i}$ and $\left(u_{p_{k}}\right)_{j} \rightarrow u_{j}$ we obtain

$$
\left|u_{i}-u_{j}\right| \leq 1 \quad \text { for }\left|x_{i}-x_{j}\right|<S
$$

Thus, we conclude that $U \in K_{\infty}$. This ends the proof.

3.2. An explicit solution. Now our goal is to show an explicit example that illustrate the behavior of the solutions when $p=+\infty$. We want to find a solution to

$$
\begin{cases}f(t)-U^{\prime}(t) \in \partial G_{\infty}(U(t)), & \text { a.e. } t \in(0, T), \\ U(0)=u_{0}\left(x_{j}\right), & \text { in } \Omega,\end{cases}
$$

where

$$
G_{\infty}(v)= \begin{cases}0 & \text { if }\left|v_{i}-v_{j}\right| \leq 1, \text { for }\left|x_{i}-x_{j}\right|<S \\ +\infty & \text { in other case. }\end{cases}
$$

In order to verify that a function $U:[0, T] \mapsto \mathbb{R}^{N+1}$ is a solution to $(3.21)$ we need to check that

$$
G_{\infty}(v) \geq G_{\infty}(U)+\left\langle f-U_{t}, v-U\right\rangle, \quad \text { for all } v \in \mathbb{R}^{N+1} .
$$

To this end we can assume that $v \in K_{\infty}$ (otherwise $G_{\infty}(v)=+\infty$ and then (3.22) becomes trivial). By (3.16), we need to check that $U(t) \in K_{\infty}$ and

$$
\sum_{j}\left(f_{j}(t)-u_{j}^{\prime}(t)\right)\left(v_{j}-u_{j}(t)\right) \leq 0
$$

for every $v \in K_{\infty}$.

Now, to simplify, assume that the support of $J$ is $(-1,1)$, that is, $S=1$. Let us consider a recipient $\Omega=(0, L)$ with $L$ an integer greater than 1 , a uniform mesh, $x_{j}=(j-1) L / N$, $j=1, \ldots, N+1$, as initial datum take $u_{0}=0$ and a source given by $f(x, t)=\chi_{[0,1]}(x)$. Then the solution is given by

$$
u_{j}(t)= \begin{cases}t, & x_{j} \leq 1 \\ 0, & x_{j}>1\end{cases}
$$

for times $t \in[0,1]$. Let us check that this is indeed the solution for $t \in[0,1]$. It is clear that $U(t) \in K_{\infty}$ since $\left|u_{j}(t)-u_{i}(t)\right| \leq 1$ for every $i, j$. Moreover, since $f$ and $U_{t}$ coincide in $[0, L]$, we have that $(3.22)$ is immediate.

For $t \in[1,3]$ we get

$$
u_{j}(t)= \begin{cases}1+\frac{t-1}{2} & x_{j} \in[0,1), \\ \frac{t-1}{2} & x_{j} \in[1,2), \\ 0 & x_{j} \notin[0,2) .\end{cases}
$$


Let us check that this is the solution. We have that $U(t) \in K_{\infty}$. Hence we have to verify (3.22). Since $U_{t}$ and $f$ vanish outside $[0,2)$ we have to see that

$$
\sum_{x_{j} \in[0,1)}\left(1-u_{j}^{\prime}(t)\right)\left(v_{j}-u_{j}(t)\right)+\sum_{x_{i} \in[1,2)}\left(-u_{i}^{\prime}(t)\right)\left(v_{i}-u_{i}(t)\right) \leq 0 .
$$

Using the explicit form of $u_{j}$ we get

$$
\sum_{x_{j} \in[0,1)} \frac{1}{2}\left(v_{j}-\left(1+\frac{t-1}{2}\right)\right)-\sum_{x_{i} \in[1,2)} \frac{1}{2}\left(v_{i}-\frac{t-1}{2}\right) \leq 0 .
$$

That is equivalent to (note that all the terms that involve $t$ cancel),

$$
\sum_{x_{j} \in[0,1)} v_{j}-\sum_{x_{i} \in[1,2)} v_{i} \leq \sum_{x_{j} \in[0,1)} 1
$$

which holds as a consequence of the fact that $\left|v_{j}-v_{i}\right| \leq 1$ for $x_{j} \in[0,1)$ and $x_{i} \in[1,2)$ (we are using here that $v \in K_{\infty}$ ).

In general we have, until the recipient is full, for any $k=1, \ldots, L$ and for $t \in\left[t_{k-1}, t_{k}\right)$

$$
u_{j}(t)= \begin{cases}k-1+\frac{t-t_{k-1}}{k} & x_{j} \in[0,1), \\ k-2+\frac{t-t_{k-1}}{k} & x_{j} \in[1,2), \\ \cdots & x_{j} \in[k-1, k), \\ \frac{t-t_{k-1}}{k} & x_{j} \notin[0, k) .\end{cases}
$$

Here $t_{k}=t_{k-1}+k$ is the first time at which the solution reaches level $k$, that is $u_{h}\left(t_{k}, 0\right)=k$.

For times even greater, $t \geq t_{L}=L(L+1) / 2$ (the recipient is full of sand), the solution turns out to be

$$
u_{j}(t)= \begin{cases}L+\frac{t-t_{L}}{L} & x_{j} \in[0,1), \\ L-1+\frac{t-t_{L}}{L} & x_{j} \in[1,2), \\ \cdots & x_{j} \in[L-1, L) . \\ 1+\frac{t-t_{L}}{L} & .\end{cases}
$$

Hence, when the recipient is full the solution grows with speed $1 / L$ uniformly in $(0, L)$.

\section{NumericAl EXPERIMENTS}

In this section we perform, using Matlab (ode15s subroutine), some numerical experiments just to illustrate our general results.

First, we show the evolution in time of a numerical solution, taking $p=5, N=101$ and as initial datum $u_{0}=6\left|\sin \left(\frac{\pi}{3} x\right)\right|+0.15(3-|x|)^{2}$ in the interval $[-3,3]$. We can 
appreciate that, since the initial datum is symmetric, the solution remains symmetric for every positive time.

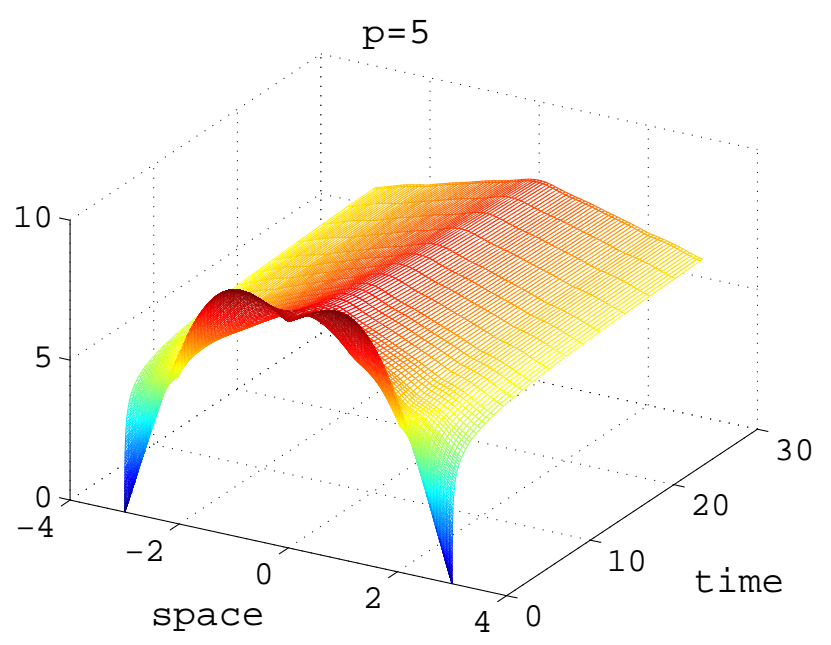

Figure 1. Evolution in time, symmetric datum.

Next, we show the value of the first node (corresponding to $x=-3$ ) of the numerical approximation for different values of $N$. We can appreciate the convergence towards the mean value of the numerical initial datum as $t$ increases for different values of $N$. Moreover, we can appreciate that the discrete mean value increases with $N$ to 6 , which is the continuous mean value of $u_{0}=9-x^{2}$.

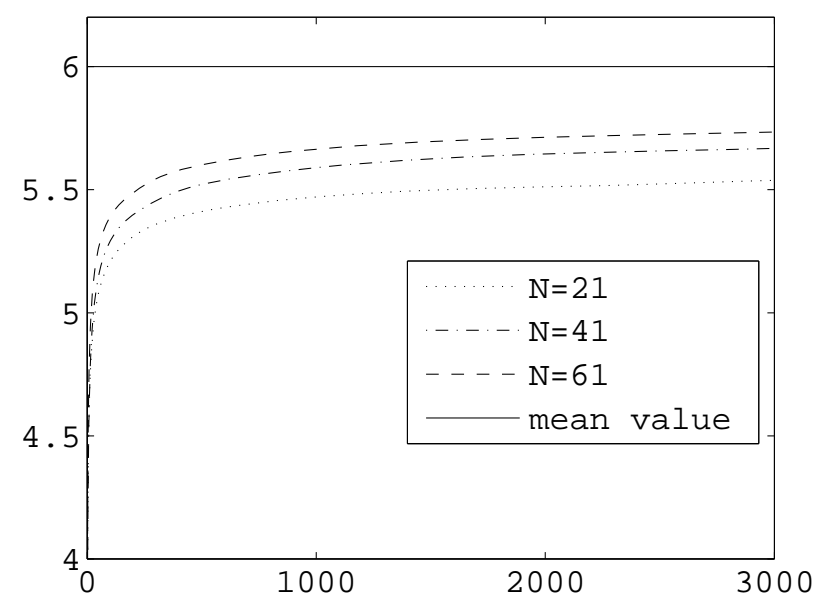

Figure 2. Convergence to the mean value of the initial datum as $t$ increases.

Now we include a picture comparing the semidiscrete solution with the totally discrete one. Here we take $p=4, u_{0}(x)=\left(9-x^{2}\right), \Omega=(-3,3), N=50, \tau_{k}=0.1$ and $k=8000$. 


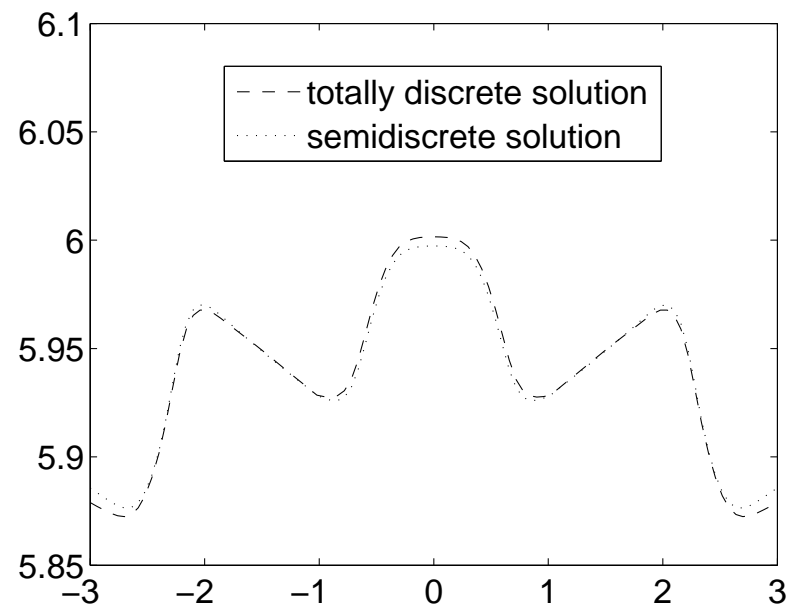

Figure 3. Semidiscrete and totally discrete solutions for $t_{k}=\tau_{k} k$.

In the next picture we show the evolution in time of a numerical model of sandpiles with $p=50$, corresponding to the initial data $u_{0}=0$ in $\Omega=(0,4)$ with a source $f=\chi_{[0,1]}$. Note that the slope of $u_{j}(t)$ decreases as time evolves, as we illustrate in Figure 5 . This is a consequence of the fact that the sand that is added by the source has to be distributed in a larger set.

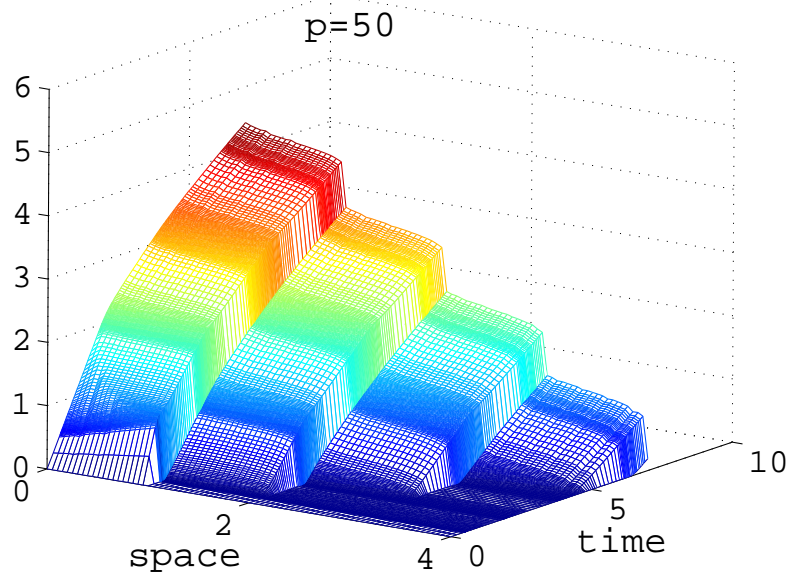

Figure 4. Evolution in time of the numerical model for sandpiles. 


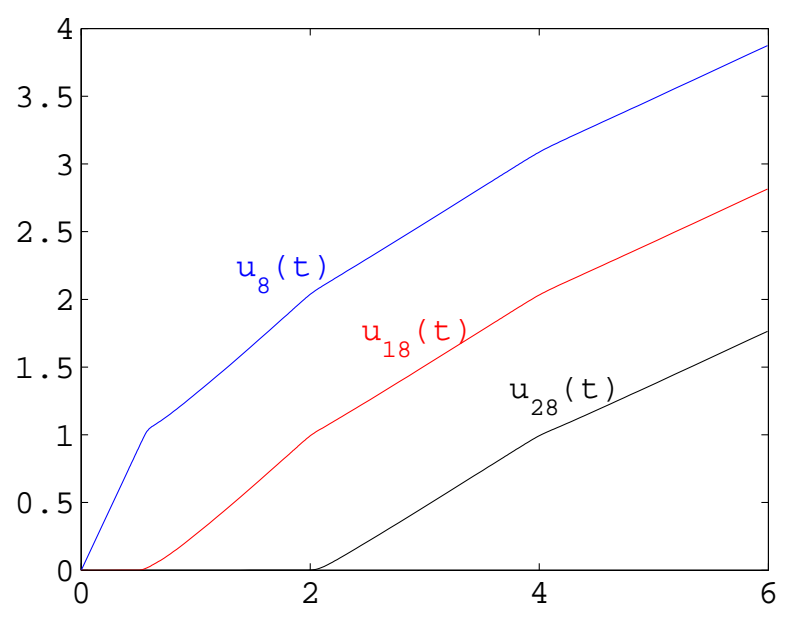

Figure 5. Different slopes of the value of the solution at some nodes of the numerical model for sandpiles.

Finally we show solutions for different values of $p$ and compare them with the explicit formula of the previous section. In this example we take $u_{0}=0$ in $\Omega=(0,3)$ with a source $f=\chi_{[0,1]}$.
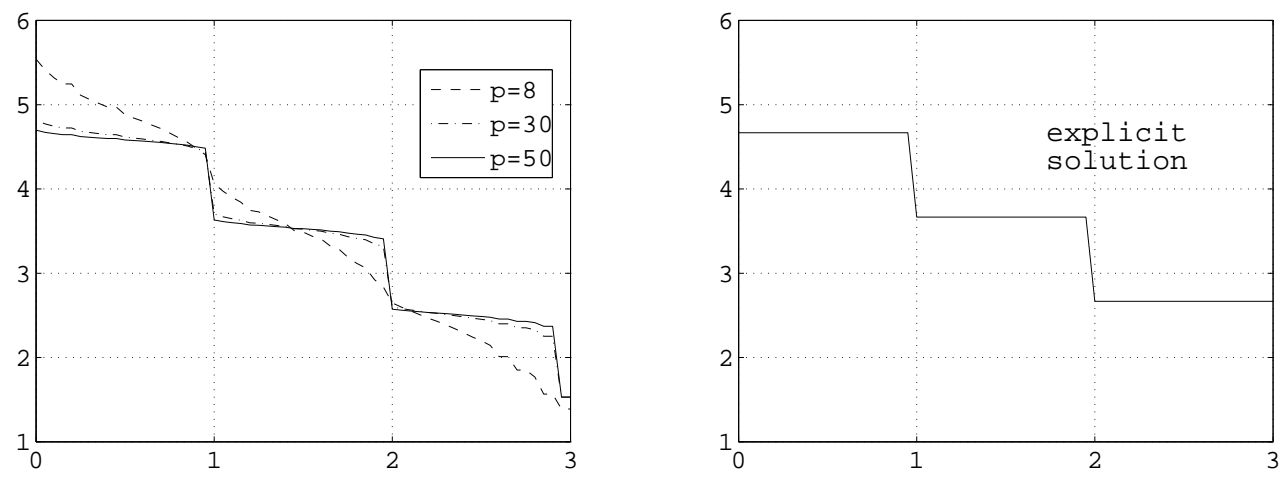

Figure 6. Convergence as $p$ increases to the explicit solution.

Acknowledgements. Part of this work was performed during a visit of MPL and JDR to Univ. de Valencia. They want to thank for the warm hospitality and the stimulating working atmosphere found there. JDR is partially supported by UBA X066 and CONICET (Argentina) and MPL by DGICYT grant MTM2005-08760-C02-01 and 02 (Spain).

\section{REFERENCES}

[1] F. Andreu, J. M. Mazón, J. D. Rossi and J. Toledo. The Neumann problem for nonlocal nonlinear diffusion equations. J. Evol. Equations. 8(1), (2008), 189-215. 
[2] F. Andreu, J. M. Mazon, J. D. Rossi and J. Toledo. The limit as $p \rightarrow \infty$ in a nonlocal $p$-Laplacian evolution equation. A nonlocal approximation of a model for sandpiles. To appear in Calc. Var. PDE.

[3] F. Andreu, J. M. Mazón, J. D. Rossi and J. Toledo. A nonlocal p-Laplacian evolution equation with Neumann boundary conditions. J. Math. Pures Appl., 90(2), (2008), 201-227.

[4] P. Bates and A. Chmaj. An integrodifferential model for phase transitions: stationary solutions in higher dimensions. J. Statistical Phys., 95, (1999), 1119-1139.

[5] P. Bates, P. Fife, X. Ren and X. Wang. Travelling waves in a convolution model for phase transitions. Arch. Rat. Mech. Anal., 138, (1997), 105-136.

[6] H. Brezis. Opérateur Maximaux Monotones et Semi-groupes de Contractions dans les Espaces de Hilbert. North-Holland, 1973.

[7] H. Brezis and A. Pazy. Convergence and approximation of semigroups of nonlinear operators in Banach spaces. J. Functional Analysis, 9 (1972), 63-74.

[8] A. Buades, B. Coll and J. M. Morel. Neighborhood filters and PDE's. Numer. Math. 150 (2006), $1-34$.

[9] C. Carrillo and P. Fife. Spatial effects in discrete generation population models. J. Math. Biol. 50(2), (2005), 161-188.

[10] E. Chasseigne, M. Chaves and J. D. Rossi. Asymptotic behaviour for nonlocal diffusion equations. J. Math. Pures Appl., 86, (2006), 271-291.

[11] X. Chen. Existence, uniqueness and asymptotic stability of travelling waves in nonlocal evolution equations. Adv. Differential Equations, 2, (1997), 125-160.

[12] C. Cortazar, M. Elgueta and J. D. Rossi. A non-local diffusion equation whose solutions develop a free boundary. Annales Henri Poincaré, 6(2), (2005), 269-281.

[13] C. Cortazar, M. Elgueta, J.D. Rossi and N. Wolanski. Boundary fluxes for non-local diffusion. J. Differential Equations, 234, (2007), 360-390.

[14] C. Cortazar, M. Elgueta, J.D. Rossi and N. Wolanski. How to approximate the heat equation with Neumann boundary conditions by nonlocal diffusion problems. Arch. Rat. Mech. Anal., 187(1), (2008), 137-156.

[15] HI. Ekeland and R. Temam. Convex Analysis and Variational Problems. North-Holland, 1972.

[16] L. C. Evans, M. Feldman and R. F. Gariepy. Fast/slow diffusion and collapsing sandpiles. J. Differential Equations, 137 (1997), 166-209.

[17] L. C. Evans and Fr. Rezakhanlou. A stochastic model for growing sandpiles and its continuum limit. Comm. Math. Phys., 197 (1998), 325-345.

[18] M. Feldman. Growth of a sandpile around an obstacle. Monge Ampère equation: applications to geometry and optimization (Deerfield Beach, FL, 1997), 55-78, Contemp. Math., 226, Amer. Math. Soc., Providence, RI, 1999.

[19] P. Fife. Some nonclassical trends in parabolic and parabolic-like evolutions. Trends in nonlinear analysis, 153-191, Springer, Berlin, 2003.

[20] P. Fife and X. Wang. A convolution model for interfacial motion: the generation and propagation of internal layers in higher space dimensions. Adv. Differential Equations, 3(1), (1998), 85-110.

[21] S. Kindermann, S. Osher and P. W. Jones. Deblurring and denoising of images by nonlocal functionals. Multiscale Model. Simul., 4, (2005), 1091-1115.

[22] U. Mosco. Convergence of convex sets and solutions of variational inequalities. Advances. Math., 3, (1969), 510-585.

[23] L. Silvestre. Hölder estimates for solutions of integro differential equations like the fractional laplace. Indiana Univ. Math. J., 55(3), (2006), 1155-1174.

[24] X. Wang. Metaestability and stability of patterns in a convolution model for phase transitions. J. Differential Equations, 183, (2002), 434-461. 
[25] L. Zhang. Existence, uniqueness and exponential stability of traveling wave solutions of some integral differential equations arising from neuronal networks. J. Differential Equations, 197(1), (2004), 162-196.

Mayte PÉREz-LLanos

Departamento de Matemáticas, U. Carlos III de Madrid, 28911

Leganés, Spain.

E-mail address: mtperez@math.uc3m.es

Julio D. Rossi

imdeA Matemáticas, C-IX, Campus Cantoblanco, UAM, (28049),

MADRID, SPAIN.

On leave from Departamento de Matemática, FCEyn UBA (1428)

Buenos Aires, Argentina.

E-mail address: jrossi@dm.uba.ar

Web page: http://mate.dm.uba.ar/ jrossi 